\title{
Blended learning en la formación permanente del profesorado. Aportaciones de asesores de formación sobre modalidades formativas
}

\section{Blended learning in the ongoing training of teachers. Contributions of the permanent training advisers on training modalities}

\author{
Antonio Paniagua \\ Universidad de Extremadura. Badajoz, España \\ apaniaguc@alumnos.unex.es \\ Ricardo Luengo \\ Universidad de Extremadura. Badajoz, España \\ rluengo@unex.es \\ José Luis Torres Carvalho \\ Universidad de Extremadura. Badajoz, España \\ jltc@unex.es \\ Luis Manuel Casas \\ Universidad de Extremadura. Badajoz, España \\ luisma@unex.es
}

\begin{abstract}
Resumen
La revisión de los sistemas educativos en España pone de manifiesto la necesidad de encontrar nuevos enfoques en la formación permanente del profesorado de los niveles previos a la Universidad. Este estudio toma la experiencia de un grupo de asesores de formación permanente para realizar un análisis cualitativo de las ventajas e inconvenientes de las modalidades de formación que se emplean tradicionalmente y definir los parámetros básicos de modalidades más acordes con las necesidades del profesorado como sucede con el proceso combinado propuesto por el paradigma de blended learning. A través de una metodología abierta, los participantes reflexionan libremente sobre estas materias, llegando a conclusiones similares a las de otros estudios realizados hasta la fecha. El tratamiento de estos relatos se realiza informáticamente para favorecer una interpretación precisa de los datos recopilados.

Palabras clave: formación del profesorado; innovación; TIC; blended learning; formación a distancia.
\end{abstract}

\begin{abstract}
The review of education systems in Spain highlights the need to find new approaches to teacher's training for pre- university levels. This study takes the experience of a group of training advisers and performs a qualitative analysis of the advantages and disadvantages of classical training methods. It defines the basic parameters according with the teachers needs with the process proposed by blended learning. By using an open methodology, participants expose their point of view on these matters freely, reaching similar conclusions to other studies reviewed. These data are processed by computer to provide an accurate interpretation of the gathered information.
\end{abstract}

Keywords: teacher's training; innovation; ICT; blended learning; online training. 


\section{1.- Introducción}

El sistema educativo español, así como el resto de los sistemas europeos, está siendo sometido a un análisis crítico constante que intenta acercarlo a las necesidades cambiantes de nuestra sociedad. Las variaciones en el modelo productivo y social, que se vienen produciendo desde la llegada de los ordenadores y de Internet, buscan su reflejo en la escuela desde hace ya más de 20 años. Más específicamente, nuestras formas de comunicarnos y relacionarnos están cambiando drásticamente, desde principios del siglo XXI, hasta conformar una sociedad del conocimiento que reclama de nuestros alumnos unas habilidades diferentes a las que se venían enseñando desde la época de la revolución industrial.

Los organismos reguladores vienen diseñando normas educativas donde los aspectos claves se centren en la adquisición de competencias más que en métodos tradicionales. Se pretende que las personas que pasan por la Escuela puedan llegar a ser ciudadanos activos y responsables, capaces de adaptarse a un modelo de sociedad aún en desarrollo. Entre esas competencias encontramos la competencia digital, recogida como un elemento clave y transversal de todos los sistemas educativos modernos. Como indica Delors (1996): "Esta revolución tecnológica constituye a todas luces un elemento esencial para entender nuestra modernidad, en la medida en que crea nuevas formas de socialización, e incluso nuevas definiciones de la identidad individual y colectiva.".

Trasladando esta cuestión al ámbito docente, observamos esta transformación de una forma clara. Autores como Prensky (2001) o Makri (2014) lo definen con claridad al expresar que la formación que tradicionalmente se venía realizando de forma presencial ha incorporado con cierta naturalidad el cambio hacia una formación a distancia aprovechando las nuevas posibilidades tecnológicas.

Ante esa realidad, cabe preguntarse qué está sucediendo con el profesorado encargado de esta tarea, ¿está realmente migrando hacia nuevos modelos de docencia o permanece resistente al cambio?

En diferentes entornos se analizan nuevas vías de trabajo y formas de interactuar con los alumnos. Así encontramos a grupos de profesores que modifican su práctica docente para asumir modelos más o menos disruptivos de trabajo. Del mismo modo, la formación del profesorado se aproxima a estas tendencias y las difunde.

A través de este estudio, desarrollado en Paniagua (2014), se profundiza sobre las tendencias actuales de formación y sobre el posicionamiento de diferentes responsables de formación del profesorado en lo referente a estas apuestas.

\section{2.- Tendencias formativas}

En España, desde el diseño del sistema de reconocimiento de actividades para el profesorado de niveles previos a la Universidad hasta la actualidad se han ido sucediendo diferentes normas. En ellas se ha ido desplazando la formación desde el nivel estatal hasta el de las Comunidades Autónomas, pero manteniendo unas estructuras básicas. Así se ha ido pasando de un modelo basado fundamentalmente en cursos de formación

Blended learning en la formación permanente del profesorado. Antonio Paniagua, Ricardo Luengo, José Luis Torres Carvalho, Luis Manuel Casas. 
presencial en sus inicios, complementados con seminarios, grupos de trabajo y jornadas cortas, a un modelo en el que la formación en centros de trabajo y a distancia ha ido cobrando protagonismo.

Hoy en día el trabajo que se realiza en formación se focaliza notablemente en esas dos vías, que se convierten casi en transversales. Encontramos numerosas acciones formativas de modalidades como seminarios o cursos, que se realizan íntegramente en el centro de trabajo de los participantes. Del mismo modo, actividades de formación a distancia se combinan de forma natural con cursos, grupos de trabajo y seminarios, conformando las nuevas tendencias en formación que pretendemos estudiar en este trabajo. Autores como Nunes y Meneses (2014) destacan la importancia de las nuevas modalidades formativas y la relevancia que están cobrando progresivamente en el día a día de la formación del profesorado.

Analizando la normativa actual podemos encontrar un cambio definitivo en este sentido en los últimos años. La orden EDU/2886/2011, BOE (2011) recoge la convocatoria y certificación de actividades de formación permanente del profesorado por parte del Ministerio de Educación, identificando las principales modalidades e incorporando una clasificación de actividades en presenciales, en red y mixtas, entendiendo ésta última como la combinación ordenada de las dos anteriores.

Otras normativas posteriores, con una visión aún más renovadora de las modalidades formativas, llevan estos cambios más allá. Por ejemplo, la Orden EDU/1057/2014. BOCYL (2014) que actualiza las modalidades formativas de la Comunidad Autónoma de Castilla y León, recoge los aspectos anteriores y otras tendencias, como los itinerarios formativos o el uso de módulos de formación en otros entornos. En el ámbito de las modalidades formativas incorpora algunas novedades como los procesos de autoformación, pero sobre todo abre el espectro a la experimentación de nuevos formatos.

Con este trasfondo, el análisis se centra en conocer las impresiones sobre la modalidad presencial y a distancia, además de estudiar cómo equilibrarlas para aprovecharlas conjuntamente.

\section{1.- Formación a distancia}

Centrándonos en la Comunidad Autónoma de Extremadura, donde se ha realizado el estudio, revisar algunos datos tomados del Registro de Formación del Profesorado para apreciar el impacto de esta formación. En él encontramos que en los últimos años se han registrado 18.390 participaciones en acciones registradas como formación a distancia. El número de actividades se ha ido incrementando progresivamente a lo largo de los años, así como su número de participantes, según los datos recabados en el registro de formación del profesorado de Extremadura. Así se ha pasado de un nivel de unas 300 participaciones anuales en los primeros años del siglo a una media de unas 2.500 participaciones anuales en los años 2010 y posteriores. Son unas cifras elevadas si consideramos que el colectivo docente afectado ronda las 11.000 personas.

En esos últimos años se ha procedido a una normalización de las actividades, mediante convocatorias fijas cada año, con el objetivo de facilitar una oferta ordenadas de formación a distancia. De este modo, se da la paradoja de que con un número menor de actividades se incrementan las participaciones registradas. Se sigue, en este formato, los

Blended learning en la formación permanente del profesorado. Antonio Paniagua, Ricardo Luengo, José Luis Torres Carvalho, Luis Manuel Casas. 
modelos diseñados por el Ministerio de Educación años atrás y empleados también por varias comunidades autónomas.

El ascenso es evidente y los motivos por los que se produce son fáciles de deducir. Como citamos en estudios anteriores (Paniagua, 2014) la formación a distancia aporta comodidad, autonomía, inmediatez, multimedia (recursos de aprendizaje variados), ahorro en los costes, acceso desde cualquier lugar y reutilización de recursos. Son ventajas tanto para el usuario como para la organización que convoca las actividades.

En conclusión, las nuevas modalidades formativas en las que se trabaje deberían respetar el máximo número de ventajas aportadas por la formación a distancia, mantenerlas y aumentarlas.

\section{2.- Formación en centro}

Aunque la formación en centro es una modalidad formativa extendida y que se podría considerar clásica, en la actualidad se utiliza de una forma masiva y cotidiana en la Comunidad Autónoma de Extremadura. Su éxito no radica sólo en la comodidad de realizarlo en su propio centro, y en un ambiente conocido, sino sobre todo porque se realiza entre compañeros de trabajo, en muchas ocasiones sin la intervención de un agente externo. Todos los estudios hasta la fecha suelen presentar a este tipo de formación como uno de las preferidas por el profesorado. Además se fomenta la identidad del propio centro, como expresa en su introducción la orden que los convoca en Extremadura (DOE, 2001): "[..] potenciando asimismo el desarrollo institucional de los propios centros como organizaciones educativas que ofrecen la mejor respuesta a los problemas socialmente relevantes y a las demandas y necesidades que la sociedad plantea en cada momento al sistema educativo".

Esta modalidad de formación en grupo, con equipos de trabajo familiares y con un objetivo claro, entronca a la perfección con tendencias actuales como el trabajo por competencias y el aprendizaje basado en proyectos.

Podemos considerar la formación a distancia y la formación en centro como las dos modalidades más demandadas por el profesorado. Son, por tanto, una base sobre la que sustentar una formación sólida y bien aceptada que satisfaga las demandas actuales.

\section{3.- Blended Learning}

El término blended learning se puede trasladar a nuestro idioma como aprendizaje combinado, aportando una idea clara de su significado. De todas las revisadas las tres definiciones más habituales empleadas (Graham, 2006) por diferentes autores son:

- Combinar modalidades formativas (o distribución de recursos).

- Combinación de métodos formativos.

- Combinación de formación presencial y a distancia.

La fecha de aparición del término, hacia 2002, se vincula claramente con uno de sus rasgos más significativos, establecido en la tercera definición: mezclar la tradición presencial con la formación a distancia. Es en esos años cuando la tecnología comienza a mostrarse lo suficiente madura como para que la formación a distancia se convierta en una realidad accesible de forma amplia.

Blended learning en la formación permanente del profesorado. Antonio Paniagua, Ricardo Luengo, José Luis Torres Carvalho, Luis Manuel Casas. 
Por tanto, Blended learning es una combinación de modalidades formativas y la acomodación de métodos de trabajo tradicionales con sistemas de formación a distancia, generando una nueva forma de aprendizaje que se ajusta a las posibilidades con las que contamos hoy en día. Es un sistema acorde con las tendencias de distribución de tiempo, espacio y relaciones interpersonales.

La efectividad del aprendizaje combinado se halla en su capacidad para unir las principales ventajas de las reuniones presenciales con la formación a distancia, asíncrona, pero además en su capacidad para generar un grupo cohesionado de trabajo según Garrison y Kanuka, 2004.

En esas ventajas hallamos también sus inconvenientes, con problemas bien identificados (Reece y Lockee, 2005) como un trabajo previo de planificación, gestión e implementación, además de concretar correctamente el programa de trabajo, y las expectativas a cumplir. Esta modalidad requiere también un mejor seguimiento del trabajo del alumnado, correctamente planificado y definido en detalle.

Podemos encontrar investigaciones cercanas sobre esta temática, como González, Rodríguez-Conde, Olmos-Migueláñez, Borham y García-Peñalvo (2013), que evalúan el impacto de la metodología blended learning desde un enfoque cuantitativo, a diferencia de otros trabajos como el presente, que se centra en una aproximación cualitativa.

Otros autores, como Bazhenov y Luchaninov (2014) o Gámiz-Sánchez y GallegoArrufat (2016) o Lim (2014) coinciden en las formas de aplicación de la metodología. En todos los casos, los estudios demuestran una mejora del interés de los usuarios y una aceptación alta de la aplicación de esta metodología, favoreciendo la autonomía del alumnado en la construcción de sus aprendizajes. En estos puntos encontramos reflejados algunos de los grandes rasgos de los sistemas educativos actuales. Vemos cómo la incorporación de las tecnologías a los procesos de enseñanza/aprendizaje incrementa la buena disposición y el interés del alumnado. Vemos también como este tipo de metodologías favorece la construcción autónoma de aprendizajes y prepara para el proceso de aprendizaje a lo largo de la vida.

Para conseguir un proceso correcto en el desarrollo de esta modalidad varios autores ponen de manifiesto la importancia de realizar un diseño bien estructurado de todos los detalles relacionados con la acción. Según Garrison y Kanuka, 2004, se debe atender al menos a una los siguientes aspectos clave:

- Desarrollo de políticas que favorezcan esta modalidad.

- Planificación estratégica: objetivos, costes y recursos disponibles.

- Planificación operativa: que incluye aspectos como estrategias de promoción, de compartición de recursos, gestión de tecnología o un adecuado proceso de evaluación.

- Recursos disponibles: donde consideramos la disponibilidad financiera, humana y técnica.

- Planificación en el tiempo: cómo implementar los cursos. Podemos centrarnos en horarios y citas fijas o por el contrario en la posibilidad de realizar sesiones flexibles cuando sean requeridas.

Blended learning en la formación permanente del profesorado. Antonio Paniagua, Ricardo Luengo, José Luis Torres Carvalho, Luis Manuel Casas. 
- Asistencia: es crítico contar con el respaldo suficiente tanto para alumnos como para formadores. En este sentido, la creación de un grupo de trabajo que respalde el proceso y que cuente con la formación apropiada resultará crucial.

Otra clave es la relación entre lo presencial y lo virtual. No es fácil determinar cuál es el balance adecuado, que obedecerá a los objetivos que se persigan y a la planificación establecida. De entre las diferentes propuestas existentes podemos encontrar algunas muy interesantes como punto de partida (Llorente, 2008), que distingue entre cinco tipos diferentes de modelos.

Coincide con esta última afirmación Turpo, 2012, que observa un proceso de evolución en la modalidad que poco a poco va desdibujando la frontera entre lo presencial y la parte a distancia para finalmente alcanzar una mezcla entre la parte tecnológica y la pedagógica.

Un diseño de blended learning representa una clara separación de la enseñanza presencial y de la enseñanza a distancia. Se trata de una reorganización de las dinámicas de enseñanza/aprendizaje comenzando con algunas necesidades contextuales y contingencias (disciplina, nivel de desarrollo y recursos). No existen dos diseños iguales de blended learning. Esto hace que la modalidad cuente con una gran complejidad. Por tanto, cada modelo requiere un estudio muy preciso y una adecuada planificación.

\section{4.- Proceso de investigación}

Conscientes de que las modalidades combinadas se encuentran cada vez más presentes en el ecosistema de la formación del profesorado, realizamos un estudio cualitativo, con un conjunto de 15 asesores de formación permanente para intentar conocer su opinión y su experiencia sobre estas cuestiones. La intención de recoger un relato lo más amplio posible de opiniones e ideas sugería que una aproximación interpretativa podría ser la más adecuada para el estudio ya que el conjunto de elementos que enmarcan el abordaje de la metodología cualitativa se constituye en un proceso capaz de organizar, orientar y sistematizar el análisis e interpretación de los datos recogidos en la investigación, tal y como afirmaba Taylor (1987).

Aunque es una metodología de investigación abierta, no resulta complicado localizar formatos de trabajo estandarizadas que garanticen un buen enfoque funcional. En este caso, se empleó el esquema que Miles y Huberman (1994) definieron y que clarifica notablemente el proceso de recogida y análisis de datos cuantitativos. Se comienza el trabajo mediante la correspondiente fase de reflexión y de revisión de literatura existente, hasta llegar a la definición de los objetivos de la investigación, para concretar y describir las actividades que se desarrollan, como indican Cubo y Luengo (2011), cuyas pautas han sido seguidas en gran medida en esta investigación: generación de hipótesis, definición operativa de variables y selección de instrumentos y del método de recogida de datos.

La definición de variables de la investigación resultó útil para articular con facilidad el manejo, organización y análisis de datos a través software de apoyo a la investigación cualitativa utilizado. Las variables que surgieron contemplan aspectos relacionados con las modalidades formativas (V1), con los asesores de formación (V2), con el profesorado (V3), con las herramientas empleadas (V4). Alrededor de estas variables se diseñaron una serie de cuestiones abiertas para favorecer el debate:

Blended learning en la formación permanente del profesorado. Antonio Paniagua, Ricardo Luengo, José Luis Torres Carvalho, Luis Manuel Casas. 


\section{V1. Modalidades formativas:}

Pregunta 1. Inconvenientes de modalidades formativas. Estamos intentando reflexionar sobre nuestra práctica como formadores y como asesores de formación. Buscamos vías para mejorar el diseño de nuestras actividades formativas. En este marco, ¿qué inconvenientes destacarías en las modalidades formativas empleadas, en concreto en los cursos presenciales y en los cursos a distancia. Destaca un par de inconvenientes.

Pregunta 2. Ventajas de modalidades formativas. Queremos reflexionar sobre las ventajas claves de la modalidad formativa presencial y a distancia. Indica algunas de las ventajas que asocies a cada modalidad. Además, ¿crees que son compatibles?

Pregunta 5. Blended Learning. Blended Learning, explicado en pocas palabras, consiste en generar acciones formativas que combinen diferentes formas de aprendizaje presencial con aprendizaje a distancia. ¿Qué te parece el concepto? ¿Qué ventajas crees que aporta respecto al resto de modalidades?

Pregunta 6. Seminarios virtuales. En concreto, en nuestra Comunidad Autónoma contamos con una modalidad parecida, que serían los seminarios virtuales, si bien éstos se basan en un modelo algo más rígido. ¿Has realizado alguna actividad similar? ¿Qué resultado obtuviste o estás obteniendo?

Pregunta 7. Características de blended learning. Si tuviésemos que definir un modelo común de blended learning para la formación del profesorado que actualmente realizamos, ¿qué características consideras que debe tener una actividad formativa en cuanto a su relación de duración y presencialidad?

V2. Gestores de formación:

Pregunta 4. Innovación en las acciones formativas. Para los que llevamos más tiempo, ¿detectas alguna innovación en modalidades formativas en los últimos años. ¿Habéis modificado las formas de diseñar acciones formativas o incorporado alguna novedad?

Pregunta 9. Organización en blended learning. ¿Qué aspectos organizativos sería necesario modificar en el diseño administrativo de una actividad basada en blended learning? ¿Y en el diseño de sus contenidos?

Pregunta 8. Modalidades mixtas. El aprendizaje combinado en ocasiones cae en una concepción errónea: tenemos un curso presencial e intentamos anexarle un bloque a distancia y viceversa. ¿Consideras que eso aporta un valor añadido al curso? Si separamos ambas partes del curso, ¿observas alguna diferencia entre ellas o podrían mantenerse como bloques autónomos? En el segundo caso, seguramente no estemos hablando de un aprendizaje combinado.

Blended learning en la formación permanente del profesorado. Antonio Paniagua, Ricardo Luengo, José Luis Torres Carvalho, Luis Manuel Casas. 
Pregunta 11. Interés de los gestores de formación. Finalmente, ¿crees que es interesante realizar este tipo de profundización en los modelos de formación o consideras que con los modelos ya existentes tenemos suficiente?

\section{V3. Profesorado:}

Pregunta 3. Interés del profesorado. En tu opinión, por lo que detectas del contacto con los usuarios, ¿cuáles son las modalidades formativas que más interesan al profesorado? ¿Por qué crees que es así?

\section{V4. Herramientas tecnológicas:}

Pregunta 10. Herramientas. ¿Qué herramientas tecnológicas emplearías para diseñar una actividad basada en blended learning?

El proceso de recogida de datos se basó en el uso de grupos focales online, atendiendo a las ventajas que expone Edmunds (1999) siendo en general muy apropiada para participantes con las características del grupo de profesionales sobre el que se realiza el trabajo: disparidad de horarios, poco tiempo disponible para encuentros presenciales y gran dispersión geográfica.

De este modo se diseñaron dos grupos que actuaron de forma asíncrona, siguiendo las pautas marcadas por Stewart (2005). Cada uno de los dos grupos de seis personas actuaron de forma independiente. Se pretendía poder contrastar las respuestas y evitar un grupo excesivamente grande, atendiendo a las consideraciones expresadas por los autores consultados.

Continuando con el proceso, desde los grupos focales online se procede a extraer los contenidos y a analizarlos. Para ello empleamos un software denominado WebQDA (Web Qualitative Data Analysis) que, según Souza y Costa (2011), facilita el trabajo colaborativo de investigadores y sirve para editar, visualizar, asociar y organizar fuentes textuales y multimedia. Permite además crear categorías, codificar, controlar, filtrar, hacer búsquedas y consultas a los datos para responder a las preguntas de investigación.

En la tabla se muestra un ejemplo de tabla generada a partir de los comentarios, contabilizando las ocurrencias de los nodos generados sobre la terminología vinculada con la primera variable estudiada.

Tabla 1. Variable Modalidades formativas

\begin{tabular}{|c|c|c|c|c|}
\hline Variable & Concepto & & & Referencias \\
\hline \multirow{11}{*}{$\begin{array}{l}\text { Modalidades } \\
\text { formativas }\end{array}$} & \multirow{6}{*}{ Modalidades clásicas } & \multirow{2}{*}{ Curso } & Inconvenientes & 28 \\
\hline & & & Ventajas & 27 \\
\hline & & \multirow{2}{*}{ Curso a distancia } & Inconvenientes & 30 \\
\hline & & & Ventajas & 27 \\
\hline & & \multirow{2}{*}{ Formación en centro } & Inconvenientes & 1 \\
\hline & & & Ventajas & 7 \\
\hline & \multirow{5}{*}{$\begin{array}{l}\text { Modalidades } \\
\text { modernas }\end{array}$} & \multirow{3}{*}{ Blended Learning } & Inconvenientes & 4 \\
\hline & & & Ventajas & 9 \\
\hline & & & Formato & 33 \\
\hline & & \multirow{2}{*}{ Seminarios virtuales } & Inconvenientes & 7 \\
\hline & & & Ventajas & 4 \\
\hline
\end{tabular}

$\overline{\text { Blended learning en la formación permanente del profesorado. Antonio Paniagua, Ricardo }}$ Luengo, José Luis Torres Carvalho, Luis Manuel Casas. 
Este tipo de tablas permite contemplar el seguimiento de las variables de forma objetiva y determinar qué aspectos han suscitado más debate o levantado más interés.

En este otro ejemplo, extraemos gracias a la herramienta informática los términos más empleados en el debate, una vez que se han eliminado las palabras vacías y aquellas que aportan poco significado.

Tabla 2. Términos más empleados

\begin{tabular}{|l|l|}
\hline Palabra & Repetición \\
\hline distancia & 96 \\
\hline presencial & 80 \\
\hline aprendizaje & 42 \\
\hline trabajo & 40 \\
\hline presenciales & 39 \\
\hline profesorado & 39 \\
\hline inconvenientes & 36 \\
\hline horas & 35 \\
\hline ventajas & 34 \\
\hline actividades & 33 \\
\hline tiempo & 33 \\
\hline docentes & 27 \\
\hline profesor & 27 \\
\hline actividad & 26 \\
\hline
\end{tabular}

Este tipo de depuración de contenidos ayuda a centrar los resultados y a articular el proceso en torno a las variables de la investigación.

\section{5.- Análisis de resultados}

A partir de los recursos generados con las fuentes recopiladas se pudo dar respuesta a los interrogantes planteados, reflejando la visión de los asesores sobre estos temas.

\section{1.- Modalidades formativas}

Analizamos en primer lugar si detectan inconvenientes en las modalidades formativas actuales para encontrar una respuesta general con los habituales problemas que nos permite deducir que los entrevistados son capaces de identificar y describir a la perfección los problemas propios de las modalidades formativas actuales, coincidiendo con los descritos por otros autores revisados.

De este modo, en el texto recopilado encontramos comentarios como este:

"[..] si nos referimos a la modalidad de cursos presenciales tal y como los solemos realizar, el inconveniente principal es tener que trabajar no solo por la mañana en el centro sino, también por la tarde en el CPR, y no una tarde sino dos, que si las unimos a la tarde que pasa en el centro el profesorado de Primaria, para la gran mayoría resulta muy dificil compaginar todo."

\section{2- Formación a distancia}

Sobre la formación a distancia los participantes identificaron también las ventajas e inconvenientes que se localizaron en marco teórico de la investigación a través de hasta 27 referencias diferentes a este tema, como:

Blended learning en la formación permanente del profesorado. Antonio Paniagua, Ricardo Luengo, José Luis Torres Carvalho, Luis Manuel Casas. 
"La ventaja de la formación a distancia es clara, tú te organizas tu tiempo y puedes distribuirlo como quieras. De nuevo ese es el inconveniente ya que como se ha comentado puede dejarse todo para el final y que no nos de tiempo."

\section{3.- Blended learning}

Tras provocar una reflexión sobre las ventajas e inconvenientes de las modalidades ya conocidas, buscábamos conocer la opinión sobre la modalidad en estudio. En este punto aparecieron dos claras tendencias en los inconvenientes, especialmente entre los participantes que ya habían realizado actividades similares. Por un lado se necesitaban ajustes normativos que amparen la modalidad y por otro se detecta una falta de cultura formativa sobre lo que supone esta modalidad. Se describe en este comentario:

Si realizamos un Seminario bajo esta modalidad, el profesorado no entiende que un seminario es algo en el que todos participamos y todos compartimos, lo entienden como un curso en el que tienes que darles las pautas y ellos hacen. Al pedirles que trabajen a distancia como sean muy espaciadas en el tiempo las sesiones presenciales se les olvida no están pendientes, al final lo acaban dejando porque trabajan de sesión presencial en sesión presencial.

Al hablar de las ventajas, hay un gran número de referencias y se insiste en que se resuelven los inconvenientes de las modalidades presenciales y a distancia, coincidiendo plenamente con lo constatado en la revisión realizada en el marco teórico.

\section{4.- Asesores de formación permanente}

Los agentes que generan la formación eran también un aspecto de sumo interés para la investigación, y sobre el que formulamos numerosas cuestiones con el objetivo principal de provocar una reflexión sobre su propio trabajo.

Entre otras cuestiones preguntamos si los asesores consideraban que habían cambiado su forma de trabajar y si detectaban innovación en su trabajo diario. De este punto sobresalen dos ideas con fuerza: la necesidad de un sistema más flexible, y de una mayor agilidad en los ámbitos normativos. Por ejemplo:

"Desgraciadamente veo poca innovación, únicamente este curso hemos conseguido que nos dejen ofertar los seminarios on line (con tres sesiones presenciales) y visto el resultado no creo que lo volvamos a ofertar."

Un detalle crucial es el de los cambios organizativos que los gestores de formación deben asumir. Esta cuestión desencadena una avalancha de propuestas organizativas para implementar esta modalidad.

Varios de los participantes ya han coordinado actividades basadas en una modalidad próxima a blended learning. Al revisar los detalles de los que se han celebrado dejan patente que los resultados no han sido los esperados, pero presentan algunas propuestas de mejora. Otro asesor lo expone así:

"La combinación de sesiones presenciales y virtuales debe ajustarse a las necesidades, expectativas y formación de los participantes.

Blended learning en la formación permanente del profesorado. Antonio Paniagua, Ricardo Luengo, José Luis Torres Carvalho, Luis Manuel Casas. 
La selección de los asistentes debe ser rigurosa y específica, ofreciendo una información previa muy clara respecto al trabajo que debe desarrollarse y teniendo en cuenta la formación previa de los solicitantes (por ejemplo, experiencia de uso de Moodle)."

O esta otra aportación:

"Pero puede ser una modalidad interesante si el profesorado que participa tiene claro en qué consisten y cuál será su papel y el del coordinador, intentando alejarlos lo máximo posible del concepto de curso a distancia."

Coinciden en sus apreciaciones con los aspectos organizativos expresados por otros autores, llegando a un nivel de concreción muy preciso, como corresponde a las inquietudes que se producen en el día a día.

\section{5.- Profesorado}

En este apartado se encuentra una postura más crítica hacia el profesorado y su nivel de compromiso, si bien se aprecian algunas ideas interesantes, como que dependiendo del nivel tecnológico de cada docente se tiende más a una modalidad u otra.

En general se destaca también que la temática debe ser atractiva y que la decisión de una modalidad u otra obedece con frecuencia a cuestiones personales. Un ejemplo:

"Para determinados momentos y tipo de aprendizaje, prefieren la modalidad presencial. Y para otros, la modalidad a distancia. Por ejemplo, el profesorado de educación física quiere formación presencial. Sin embargo cuando se trata de una formación TIC o incluso de idiomas, pero no nivel inicial, prefieren a distancia."

\section{6.- Herramientas tecnológicas}

La tradición de trabajo de los asesores con Moodle y su relación cotidiana con ella hizo que la mayor parte de las propuestas se encaminasen a esta opción, complementándola con recursos propios de la web 2.0 más vinculados al trabajo colaborativo y social. Este comentario lo resume:

"Yo también apostaría Moodle, complementándolo con muchas de las herramientas que habéis citado e integrando en la medida de lo posible los contenidos en el curso elaborados desde otras aplicaciones gracias al formato SCORM, utilizando videconferencias con BigBlueButton, Talleres con evaluación por rúbricas... Las posibilidades de Moodle son enormes y creo que normalmente no las aprovechamos lo suficiente."

\section{7.- Administración educativa}

Por último, los participantes insistían recurrentemente en la necesidad de clarificar mediante normativa las funciones y las posibilidades de desarrollo de su trabajo.

Hemos visto en el marco teórico que otras comunidades autónomas ya están emprendiendo este camino de modernizar las estructuras de formación para facilitar aprendizajes combinados, por lo que esta percepción de los participantes es claramente acertada.

Blended learning en la formación permanente del profesorado. Antonio Paniagua, Ricardo Luengo, José Luis Torres Carvalho, Luis Manuel Casas. 


\section{6.- Discusión de resultados}

En general los resultados obtenidos coinciden con los de la bibliografía consultada. Se valoran positivamente las metodologías combinadas, como indicaban Marki (2014) y se localizan las mismas fortalezas y debilidades y aspectos organizativos contemplados por el resto de los autores, como Reece y Lockee (2005) o Gámiz-Sánchez y Gallego-Arrufat (2016).

Entre las diferencias observadas con algunos autores que han constituido referentes en nuestro marco teórico cabe destacar la poca referencia al proceso de evaluación y certificación de las actividades basadas en la modalidad blended learning, que sí se destaca por parte de autores como Bazhenov y Luchaninov (2014). Este aspecto forma parte de la cultura habitual de los asesores consultados, por lo que quizás la diferencia estriba únicamente en que no se les consultó explícitamente sobre ello, dando por entendido que es una fase más del proceso formativo.

Las necesidades de una revisión de normativa que permita acoger los cambios en modalidades ha sido puesto de manifiesto por los asesores y encuentra fiel reflejo en los estudios que se están publicando en este sentido y que hemos revisado anteriormente, Boletín Oficial del Estado (2011) y Comunidad de Castilla y León (2014). Coinciden también en este ámbito con Nunez y Meneses (2014) al localizar soluciones eficaces. Contamos con propuestas, por tanto, que incorporan modalidades a distancia y que permiten su combinación con frases presenciales de un mínimo de 10 horas, postura acorde con lo expresado por los participantes en el estudio.

\section{7.- Conclusiones}

Del proceso del estudio se llega a la conclusión de que los participantes en el estudio, llegan a conclusiones similares a las de los estudios revisados en el marco teórico y valoran adecuadamente tanto las ventajas que aporta la modalidad estudiada como los riesgos que entraña su implantación.

Como puntos fuertes del trabajo realizado consideramos que se ha provocado una revisión de los métodos de trabajo y una reflexión sobre cómo se está desarrollando su práctica profesional en la profundización hacia nuevas metodologías de enseñanza/aprendizaje.

En lo que se refiere específicamente a blended learning, se produce una respuesta unánime que deriva del análisis de ventajas e inconvenientes de las modalidades formativas existentes: si las modalidades presenciales y las a distancia tienen inconvenientes y ventajas, éstos se eliminarán al fusionar las dos modalidades usando las ventajas de cada una de ellas.

El segundo factor común consiste en reclamar a la administración la flexibilidad necesaria para que se puedan producir este tipo de actividades.

Estamos, por tanto, ante una tendencia que ya se recoge en los planes de formación del profesorado de forma oficiosa y que poco a poco formará parte del día a día del sistema educativo como una vía más para un cómodo acceso al perfeccionamiento docente.

Como limitación de la investigación se pone de manifiesto que se debe profundizar en tanto en los aspectos relacionados con la evaluación y la certificación de actividades

Blended learning en la formación permanente del profesorado. Antonio Paniagua, Ricardo Luengo, José Luis Torres Carvalho, Luis Manuel Casas. 
como en lo que se refiere a las tipologías internas del desarrollo de la modalidad que deberían ser fruto de estudio en posteriores trabajos. Son aspectos tratados en otros trabajos analizados que no parecen resultar relevantes para los asesores de formación permanente en su relación con esta modalidad, mientras que en el resto de modalidades sí son muy importantes.

Presentación del artículo: 3 de marzo de 2016.

Fecha de aprobación: 15 de abril de 2016.

Fecha de publicación: 30 de enero de 2017.

Paniagua, A., Luengo, R., Torres Carvalho, J.L., y Casas, L.M. (2017). Blended learning en la formación permanente del profesorado. RED. Revista de Educación a Distancia, 52. Consultado el (dd/mm/aaaa) en http://www.um.es/ead/red/52

\section{Referencias bibliográficas}

Bazhenov, R. I., \& Luchaninov, D. V. (2014). Use of blended learning elements for formation of a humanitarian student's creative initiative at learning modern information technologies. Life Science Journal, 11(11s), 371-374.

Boletín Oficial del Estado (2011). Orden EDU/2886/2011, de 20 de de octubre, por la que se regula la convocatori, reconocimeinto, certificaicón y registro de las actividades de formación permanente del profesorado. $\mathrm{BOE} \mathrm{n}^{\mathrm{o}} 260$ de 28 de octubre de 2011. Disponible en http://www.boe.es/diario_boe/txt.php?id=BOE-A2011-16923

Comunidad de Castilla y León (2014). ORDEN EDU/1057/2014, de 4 de diciembre, por la que se regulan las modalidades, convocatoria, reconocimiento, certificación y registro de las actividades de formación permanente del profesorado de enseñanzas no universitarias que presta sus servicios en centros docentes sostenidos con fondos públicos en la Comunidad de Castilla y León organizadas por la Red de formación y se establecen las condiciones de reconocimiento de las actividades de formación organizadas por otras entidades. Disponible en http://bocyl.jcyl.es/boletines/2014/12/16/pdf/BOCYL-D-16122014-2.pdf

Cubo Delgado, S., \& Luengo González, R. (2011). El proceso de planificación de la investigación. En S. Cubo Delgado, B. Martín Marín, \& J. L. García Ramos (Eds.), Métodos de investigación y análisis de datos en ciencias sociales y de la salud (pp. 45-89). Madrid: Ediciones Pirámide.

Edmunds, H. (1999). The focus group research handbook. The Bottom Line, 12(3), 4646.

Gámiz Sánchez, V., \& Gallego Arrufat, M. J. (2016). Modelo de análisis de metodologías didácticas semipresenciales blended learning en educación superior. Educación XX1, 19(1), 39-61. doi:10.5944/educXX1.13946

Garrison, D. R., \& Kanuka, H. (2004). Blended learning: Uncovering its transformative potential in higher education. The Internet and Higher Education, 7(2), 95-105.

Blended learning en la formación permanente del profesorado. Antonio Paniagua, Ricardo Luengo, José Luis Torres Carvalho, Luis Manuel Casas. 
González, A. B., Rodríguez-Conde, M. J., Olmos-Migueláñez, S., Borham, M., \& García-Peñalvo, F. J. (2013). Experimental evaluation of the impact of b-learning methodologies on engineering students in Spain. Computers in Human Behavior, 29(2), 370-377. doi:10.1016/j.chb.2012.02.003.

Graham, C. R. (2006). Blended learning systems. C. J. Bonk \& C. R. Graham (Eds.), The handbook of blended learning: Global perspectives, local designs. Pfeiffer.

International Commission on Education for the Twenty-first Century, \& Delors, J. (1996). La Educación encierra un Tesoro: Informe a la UNESCO de la Comisión Internacional sobre la Educación para el Siglo XXI. Compendio. Santillana.

Junta de Extremadura (2011). ORDEN de 12 de mayo de 2011 por la que se convocan ayudas para la realización de proyectos de formación en centros educativos para el curso 2011/2012. DOE $\mathrm{n}^{\circ} 99$ de 25 de mayo de 2011. Disponible en http://doe.juntaex.es/pdfs/doe/2011/990o/11050174.pdf

Lim, D. H., Morris, M. L., \& Kupritz, V. W. (2007). Online vs. Blended Learning: Differences in Instructional Outcomes and Learner Satisfaction. Journal of Asynchronous Learning Networks, 11(2), 27-42.

Llorente Cejudo, M. D. C. (2008). Blended learning para el aprendizaje en nuevas tecnologías aplicadas a la educación: un estudio de caso. Doctoral dissertation, Tesis doctoral inédita. Sevilla, Universidad de Sevilla.

Makri, K., Papanikolaou, K., Tsakiri, A., \& Karkanis, S. (2014). Blending the Community of Inquiry Framework with Learning by Design: Towards a Synthesis for Blended Learning in Teacher Training. Electronic Journal of e-Learning, 12.

Miles, M. B., \& Huberman, A. M. (1994). Qualitative data analysis: An expanded sourcebook. Thounsand Oaks, California: Sage.

Nunes, R, Meneses, A (2014) Facebook's contribution in the process of collaborative learning. In Libro de Actas de " 3 " Congreso Ibero-Americano en Investigación Cualitativa” (Vol. 1, p. 62).

Paniagua, A. (2014). Tendencias en modalidades formativas. Blended learning. TFM. Departamento de Didáctica de las CC Experimentales y de las Matemáticas. Universidad de Extremadura.

Prensky, M. (2001). Digital natives, digital immigrants part 1. On the horizon, 9(5), 1-6.

Reece, M., \& Lockee, B. (2005). Improving training outcomes through blended learning. Journal of Asynchronous Learning Networks, 9(4), 49-57.

Souza, F. N., Costa, A. P., \& Moreira, A. (2011). Análise de Dados Qualitativos Suportada pelo Software WebQDA. En Atas da VII Conferência Internacional de TIC na Educação: Perspetivas de Inovação (pp. 49-56).

Stewart, K., \& Williams, M. (2005). Researching online populations: the use of online focus groups for social research. Qualitative Research, 5(4), 395-416.

Blended learning en la formación permanente del profesorado. Antonio Paniagua, Ricardo Luengo, José Luis Torres Carvalho, Luis Manuel Casas. 
Taylor, S. J., \& Bogdan, R. (1987). Introducción a los métodos cualitativos de investigación. La Búsqueda de Significados. Barcelona: Paidós.

Turpo Gebera, O. (2013). Perspectiva de la convergencia pedagógica y tecnológica en la modalidad blended learning. RED. Revista de Educación a Distancia, 39. 15 de diciembre de 2013. 